\title{
Validating under-resolved turbulence intensities for PIV experiments in canonical wall-bounded turbulence
}

\author{
J. H. Lee - Kevin - J. P. Monty · N. Hutchins
}

Received: date / Accepted: date

\begin{abstract}
The discrepancy between measured turbulence intensity obtained from experiments in wall bounded turbulence and the fully-resolved reference results (usually from DNS datasets) are often attributed to spatial resolution issues, especially in PIV measurements due to the presence of spatial averaging within the interrogation region/volume. In many cases, in particular at high Reynolds numbers (where there is a lack of DNS data), there is no attempt to verify that this is the case. There is a risk that attributing unexpected PIV statistics to spatial resolution, without careful checks, could mask wider problems with the experimental setup or test facility. Here we propose a robust technique to validate the under-resolved PIV obtained turbulenceintensity profiles for canonical wall-bounded turbulence. This validation scheme is independent of Reynolds number and does not rely on empirical functions. It is based on arguments that (i) the viscous-scaled small-scale turbulence energy is invariant with Reynolds number and that (ii) the spatially under-resolved measurement is sufficient to capture the large-scale energy. This then suggests that we can estimate the missing energy from volume-filtered DNS data at much lower Reynolds numbers. Good agreement is found between the experimental results and estimation profiles for all three velocity components, demonstrating that the estimation tool successfully computes the missing energy for given spatial resolutions over a wide range of Reynolds numbers. A database for a canonical turbulent boundary layer and associated MATLAB function are provided that enable this missing energy to be calculated across a
\end{abstract}

J. H. Lee (凶) · Kevin · J. P. Monty · N. Hutchins Department of Mechanical Engineering, University of Melbourne, Parkville, VIC 3010, Australia E-mail: jung.lee@unimelb.edu.au range of interrogation volume sizes, so that users do not require access to raw DNS data. This methodology and tool will provide PIV practitioners, investigating canonical wall-bounded turbulent flow with a convenient check of the effects of spatial resolution on a given experiment.

\section{Introduction}

Spatial attenuation in turbulence measurements due to the limited resolvable scale is a well-known issue in measurements of wall-bounded turbulence. The energy contribution from smaller-scale eddies is often underresolved in experiments, causing an underestimation of the measured turbulence intensities. This problem arises because of the finite effective sensor size. For hotwire anemometry (HWA) the averaging occurs along the wire length, and for particle image velocimetry (PIV) the averaging occurs within the interrogation volume (Adrian and Westerweel, 2011). Extensive reviews on spatial resolution effects have been performed for the HWA (Johansson and Alfredsson, 1983; Ligrani and Bradshaw, 1987; Hutchins et al, 2009; Ng et al, 2011) as well as using direct numerical simulations (DNS) (Philip et al, 2013b,a). Since PIV has become an important tool in turbulence research, the effects of volumetric averaging on the measured turbulence statistics have also been assessed and reported in more recent years (Kähler et al, 2006). Segalini et al (2014) have recently suggested corrections for coarse-resolution PIV. They provided a theoretical model which estimates the effect of an interrogation region in measured statistics based on an assumption that flows are homogeneous within the interrogation region. It has also been shown that applying a spatial filter on DNS data at a matched 
Reynolds number to experiments simulates the PIV spatial resolution effects quite well (Saikrishnan et al, 2006; Lavoie et al, 2007; Worth et al, 2010; Buxton et al, 2011; de Silva et al, 2012). It is important to note that these findings are obtained by directly comparing statistics of experiments and DNS data at an equivalent Reynolds number, and hence would not currently be an option for high Reynolds number experiments.

Many researchers have attempted to formulate correction schemes for the streamwise turbulence intensity acquired in under-resolved experiments. One of the widely used correction schemes for HWA measurements is that proposed by Wyngaard (1968), which is based on the assumption of isotropic turbulence. However, wall-bounded flows are strongly anisotropic in the nearwall region. Following the development of DNS , Suzuki and Kasagi (1992) investigated the effect of spatial resolution for $\times$-wire probes utilising DNS channel flow and proposed a correction method based on the twopoint correlation functions. More recently, Segalini et al (2011) proposed a correction scheme using two single hot-wire probes of different lengths required to compute the two-point correlation function of the streamwise velocity component. This technique is applicable in any turbulent flow when the flow is statistically homogeneous in the wire-length direction. Chin et al (2011) used a DNS dataset of a fully developed channel flow data to examine the spatial attenuation effects of HWA on streamwise turbulence intensity and energy spectra. They proposed an empirical missing energy model from the two-dimensional spectra. Further to this, Smits et al (2011) have presented a correction scheme on the basis of the attached eddy hypothesis. The correction provides a functional form for the unresolved streamwise turbulence intensity when spatially averaged in the spanwise direction due to sensor size of a single-normal hotwire.

In this study, instead of proposing a further correction scheme, we attempt to provide a diagnostic tool to estimate the amount of missing energy of experimental data over a wide range of Reynolds numbers. This can serve as a useful validation tool for checking that the under-resolved PIV measurements are measuring accurate statistics, when one accounts for spatial resolution effects. This forms a verification that the PIV experiment is not beset by further problems. Throughout this paper, $x, y$ and $z$ represent the streamwise, spanwise and wall-normal directions, with $u, v$ and $w$ denoting the fluctuating velocity components, respectively. Overbars (e.g. ${\overline{u^{2}}}^{+}$) and capitalised velocities (e.g. $U$ ) indicate time-averaged quantities. The superscript ' + ' is used to denote quantities normalised by viscous scaling
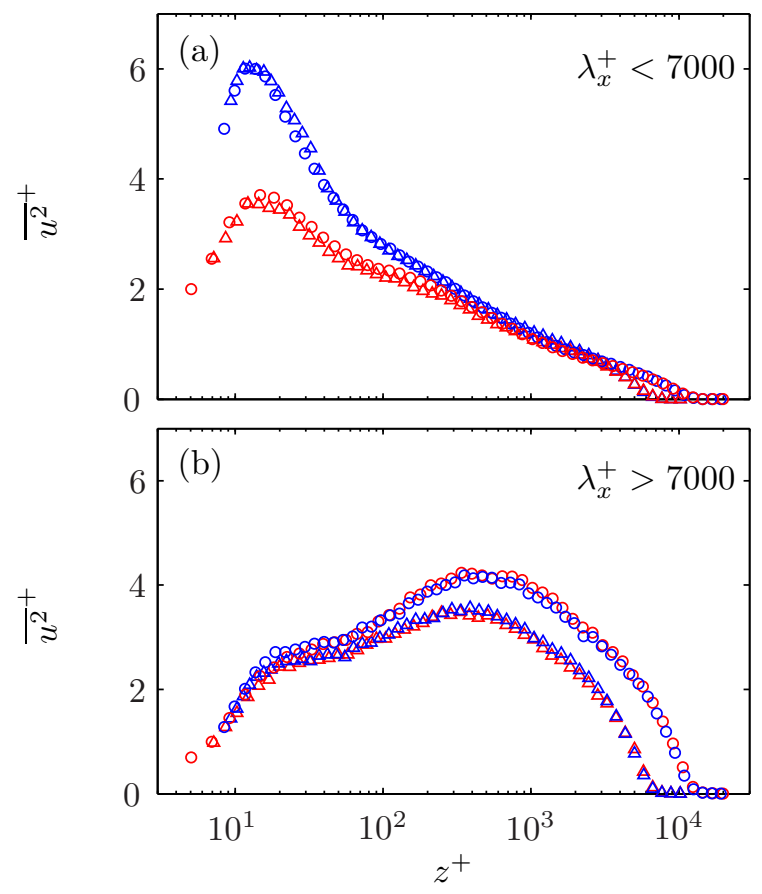

Fig. 1: Inner-scaled streamwise turbulence intensity profiles decomposed into (a) small-scale $\left(\lambda_{x}^{+}<7000\right)$ and (b) large-scale components $\left(\lambda_{x}^{+}>7000\right)$ for two different Reynolds numbers $R e_{\tau} \approx 7300$ and 14000. $\lambda_{x}^{+}$ is the inner-scaled streamwise wavelength of Fourierdecomposed fluctuations. $\triangle$, $\circ$ represent $R e_{\tau} \approx 7300$ and 14000 respectively. The blue and red symbols indicate the turbulence intensity profiles measured with inner-scaled hot-wire lengths of $l^{+}=22$ and $l^{+}=79$ respectively. Reproduced from Hutchins et al (2009)(figure 7$)$.

(e.g. $z^{+}=z U_{\tau} / \nu$ and $U^{+}=U / U_{\tau}$, where $U_{\tau}$ is the friction velocity and $\nu$ is the kinematic viscosity.)

\section{Estimation technique}

In addressing the reason for the near-wall peak scatter of pre-existing HWA data, Hutchins et al (2009) decomposed the streamwise turbulence intensity into contributions from the small- and large-scale fluctuations. This was performed for two different Reynolds numbers $R e_{\tau} \approx 7300$ and 14000 , and also with two matched inner-scaled hot-wire lengths $\left(l^{+}=l U_{\tau} / \nu=\right.$ 22 and 79 , where $l$ is the wire length). These decomposed streamwise turbulence intensity profiles are reproduced from Hutchins et al (2009) and shown in figure 1 . The symbol shapes denote Reynolds number (triangles for $R e_{\tau}=7300$ and circles for $R e_{\tau}=14000$ ), and the colour denotes $l^{+}$(blue for $l^{+}=22$ and red for $\left.l^{+}=79\right)$. Figure 1(a) highlights that the measured 
small-scale energy is invariant with Reynolds number. The $l^{+}=22$ wire (blue symbol) measures the same inner-scaled small-scale contribution for $R e_{\tau} \approx 7300$ $(\triangle)$ and $14000(\circ)$. The $l^{+}=79$ wire (red symbol) also measures the same small-scale contribution for both Reynolds numbers, albeit at a more attenuated energy level than the smaller wire length. The results indicate that the inner-scaled small-scale energy contributions are invariant with Reynolds numbers, and are only affected by an inner-scaled spatial resolution. On the other hand, the large-scale contributions shown in figure $1(b)$ seem to increase with Reynolds number and are unaffected by spatial resolution. These observations from Hutchins et al (2009) are important, since this invariance of inner-scaled small-scale contribution with Reynolds number allows us to apply the missing energy obtained from a lower Reynolds number DNS dataset to higher Reynolds number flows. The suggestion here is that the Reynolds number trend is predominantly contained within the large-scale energy, which within reason, will be relatively immune to issues of spatial resolution. As a result, one can surmise that the amount of spatial attenuation for a given interrogation volume in a PIV measurement at high Reynolds number, could be estimated by simply calculating the missing smallscale energy associated with the same interrogation volume as evaluated from a lower Reynolds number DNS dataset. This is the premise for the current method. Though figure 1 only shows this Reynolds number invariance of small-scale energy for the $u$ component, recent results from Baidya (2016) indicate that the same is also true for $v$ and $w$ components, and as such the proposed scheme should be capable of estimating missing small-scale energy for all three components of Reynolds stress. As an additional note, figure 1 and the results of Baidya (2016) are for a developing zero-pressuregradient turbulent boundary layer, however $\mathrm{Ng}$ et al (2011) have shown a similar result for turbulent channel flows over a range of Reynolds number $\left(1000 \lesssim R e_{\tau} \lesssim\right.$ 3000 ), indicating that the current estimation scheme could be extended to internal geometries.

It is important to note from figure $1(a)$ that there are small differences between the small scale energy for the two Reynolds numbers at matched $l^{+}$for $z / \delta>0.5$. This would suggest that missing energy evaluated from a DNS database at say $R e_{\tau}=1000$, could only be accurately applied at higher Reynolds numbers for $z^{+} \lesssim$ 500. However, again a cursory inspection of the difference between the $l^{+}=22$ and ${ }^{+}=79$ data (blue and red data) on figure $1(a)$, suggests that realistic spatial resolutions, the missing energy would be rather small at this wall-normal location.

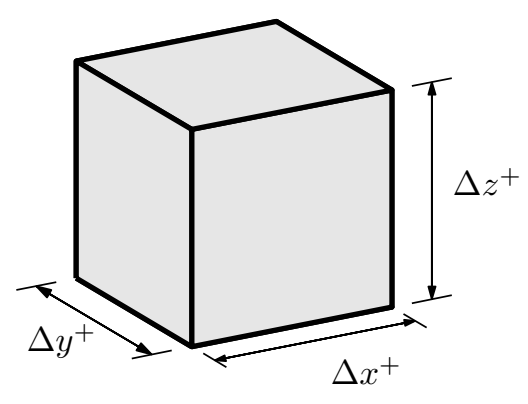

Fig. 2: A schematic of a spatial volume filter applied on DNS volumes. Here, $\Delta x^{+} \times \Delta z^{+}$and $\Delta y^{+}$are used to simulate the interrogation window and laser sheet of a PIV experiment, respectively.

Here we use the Reynolds number invariance argument of the inner-scaled small-scale energy to estimate the amount of attenuation in the Reynolds stresses due to a given interrogation volume. The method of estimating the attenuation due to the spatial averaging and how it is used to validate experimental results is described in the following steps and in figures 3 and 4 using the streamwise velocity component ${\overline{u^{2}}}^{+}$. However, it is noted that the proposed correction will be the same for the other components of fluctuating velocity. A turbulent boundary layer DNS field of Sillero et al (2014) at $R e_{\tau} \approx 1500$ is employed to simulate the influence of spatial resolution.

- Step 1: The low Reynolds number turbulent boundary layer DNS fields are spatially volume filtered at the resolution of the experimental data $\Delta x^{+} \times$ $\Delta y^{+} \times \Delta z^{+}$. A schematic of the volume filter applied on the DNS datasets is shown in figure 2. The inner-scaled turbulence intensity profiles are then computed from these filtered fields. Figure $3(a)$ and (b) illustrate this step, showing the $u$ variance profiles for the unfiltered DNS $\left(\left.\bar{u}^{2}\right|_{D N S}\right)$, and for the DNS filtered at the chosen interrogation volume size $\left(\left.{\overline{u^{2}}}^{+}\right|_{D N S_{f}}\right)$ respectively.

- Step 2: Subtracting the turbulence intensity profile of the filtered fields from the original DNS profile gives the missing small-scale energy owing to spatial resolution,

$$
\left.\Delta{\overline{u^{2}}}^{+}\right|_{S R^{+}}=\left.{\overline{u^{2}}}^{+}\right|_{D N S}-\left.\overline{u^{2}}\right|_{D N S_{f}}
$$

where $\left.\Delta{\overline{u^{2}}}^{+}\right|_{S R^{+}}$is the 'unresolved' small-scale contribution, which is universal with Reynolds number for a given inner-scaled spatial resolution. This process is illustrated in figure 3 , with $\left.\Delta{\overline{u^{2}}}^{+}\right|_{S R^{+}}$shown 


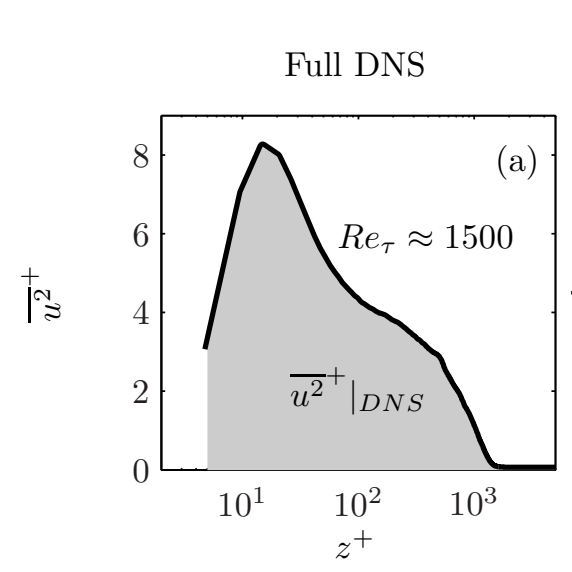

Filtered DNS at

$\Delta x^{+} \times \Delta y^{+} \times \Delta z^{+}$
Missing energy = Full DNS - Filtered DNS

Fig. 3: Missing energy estimation method. (a) Fully resolved DNS statistic. (b) Statistic from filtered DNS fields. (c) Missing small-scale energy $\left.\Delta{\overline{u^{2}}}^{+}\right|_{S R^{+}}$due to inner-scaled spatial resolution.
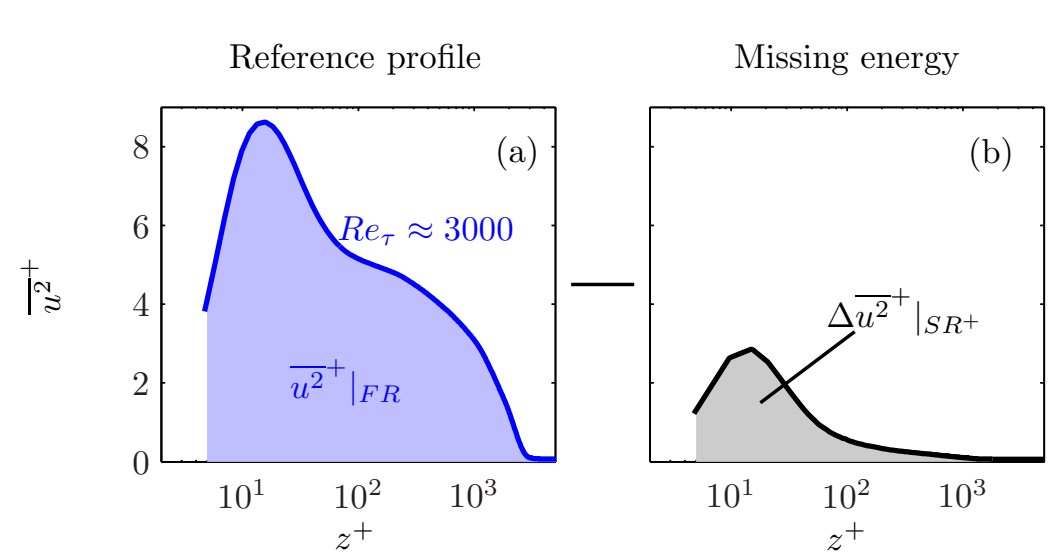

Estimated profile $=$ Reference profile - Missing energy

Fig. 4: Estimating 'attenuated' turbulence intensity profile at a higher Reynolds number using previously computed missing energy in figure 3. (a) Well-resolved reference statistic of $R e_{\tau} \approx 3000$. (b) $\left.\Delta{\overline{u^{2}}}^{+}\right|_{S R^{+}}$calculated from $R e_{\tau} \approx 1500$. (c) Comparing the 'under-resolved' statistic (dashed line) against the fully-resolved statistic (solid line).

in plot $(c)$.

- Step 3: The missing small-scale energy $\left.\Delta{\overline{u^{2}}}^{+}\right|_{S R^{+}}$ computed from the lower Reynolds number DNS data can then be subtracted from a reference turbulence intensity profile $\left(\left.{\overline{u^{2}}}^{+}\right|_{F R}\right)$ at a different (in this case higher) Reynolds number to predict the variance profile that would be measured with the given spatial resolution.

$$
\left.{\overline{u^{2}}}^{+}\right|_{E s t}=\left.\overline{u^{2}}\right|_{F R}-\left.\Delta{\overline{u^{2}}}^{+}\right|_{S R^{+}},
$$

where $\left.{\overline{u^{2}}}^{+}\right|_{E s t}$ is the predicted or estimated profile with a spatial resolution of $\Delta x^{+} \times \Delta y^{+} \times \Delta z^{+}$. Figure 4 uses the fully-resolved statistics $\overline{u^{2}}{ }_{F R}$ from Eitel-Amor et al (2014) at $R e_{\tau} \approx 3000$, and then uses the missing energy $\left.\Delta{\overline{u^{2}}}^{+}\right|_{S R^{+}}$calculated at $R e_{\tau}=1500$, to simulate the expected spatial attenuation at $R e_{\tau}=3000$ due to the given resolution. A comparison between the well-resolved profile (solid line) and the 'attenuated' estimation profile (dashed line) is shown in figure 4(c).

\subsection{Estimation for missing ${\overline{v^{2}}}^{+}$and ${\overline{w^{2}}}^{+}$}

The current technique is based on the two observations from Hutchins et al (2009), namely that (i) there is a Reynolds number invariance of small-scale energy and (ii) that within reasonable limits spatial resolution will only effect the small-scales, and that even a spatially attenuated measurement is sufficient to capture 
the large-scale (Reynolds number dependent) information. In Hutchins et al (2009), this was only demonstrated for the $u$ component. More recently, Baidya (2016) has shown that the same arguments hold for the $v$ and $w$ components. Baidya (2016) uses $\times$-wires to cover the Reynolds number range $2500 \lesssim R e_{\tau} \lesssim 10000$ with matched inner-scaled sensor length $\left(l^{+} \approx 15\right)$, measuring all three velocity components. Using a similar large- and small-scale decomposition to Hutchins et al (2009), it is demonstrated that the $v$ and $w$ components exhibit similar trends (small-scale invariance with Reynolds numbers, and Reynolds number trend captured by large-scale). These observations suggest that the estimation technique proposed above for missing ${\overline{u^{2}}}^{+}$can be extended to ${\overline{v^{2}}}^{+}$and ${\overline{w^{2}}}^{+}$. It is also likely that this technique can be expanded to the Reynolds shear stress $u w$, since the $u w$ co-spectra also appear to exhibit a small-scale invariance with Reynolds numbers (Chin et al, 2014; Baidya, 2016). However, at the present time we lack a well-resolved $\overline{u w}^{+}$reference at high Reynolds number to confirm this argument.

The novelty of the current technique is that it provides missing energy profiles of a given inner-scaled spatial resolution (which could be one, two or three dimensional averaging), for all three velocity components across a wide range of Reynolds numbers. A comparison of the turbulence intensity profiles between the attenuated experimental results and the 'attenuated' estimation profiles can be made, indicating the validity of the PIV measured statistics. Certainly this comparison can be used to assess whether the attenuation observed in the turbulence intensity profiles of the experimental results is caused by the spatial averaging or other experimental uncertainties such as issues with the test facility or experimental set-up. The technique described in this section utilises a canonical turbulent boundary layer as an example. However, the current method is also applicable for internal geometries such as pipes and channels, to obtain the missing small-scale energy of the internal flows.

\section{Experiment and Discussion}

To test the aforementioned estimation method, we use PIV datasets from towed plate experiments performed in a tow tank facility at the University of Melbourne. A $5.0 \mathrm{~m}$ long flat plate is towed at $1 \mathrm{~m} / \mathrm{s}$ using a fully automated traversing carriage. A spatially developing turbulent boundary layer is formed on the flat plate which is towed past a stationary PIV system which measures instantaneous streamwise-wall-normal plane of data with Reynolds numbers in the range of $900 \lesssim$

\begin{tabular}{|c|c|c|c|c|c|}
\hline \multicolumn{4}{|c|}{$\begin{array}{l}\text { Plate velocity }\left(U_{\text {plate }}\right) \\
\text { Laser sheet thickness }\end{array}$} & \multicolumn{2}{|c|}{$\begin{array}{l}1.0 \mathrm{~m} / \mathrm{s} \\
1.5 \mathrm{~mm} \\
\text { units })\end{array}(\approx 50$ viscous } \\
\hline & $x_{\text {plate }}$ & $R e_{\tau}$ & $\delta$ & \multicolumn{2}{|c|}{ Interrogation window size } \\
\hline & $(\mathrm{m})$ & & $(\mathrm{mm})$ & Pixel & Viscous units \\
\hline$\square$ & 0.5 & 890 & 22 & $32 \times 32$ & $50 \times 50$ \\
\hline$\nabla$ & 1.5 & 1556 & 41 & $32 \times 32$ & $47 \times 47$ \\
\hline$\circ$ & 2.5 & 2092 & 57 & $32 \times 32$ & $46 \times 46$ \\
\hline$\triangle$ & 3.5 & 2589 & 73 & $32 \times 32$ & $44 \times 44$ \\
\hline
\end{tabular}

Table 1: Experimental parameters for the towed plate experiment.

$R e_{\tau} \lesssim 2500$. Table 1 provides experimental parameters. $U_{\text {plate }}$ is the plate velocity and $x_{\text {plate }}$ is the streamwise distance downstream of the trip device. Both of $U_{\tau}$ and $\delta$ are determined from the composite velocity profile of Chauhan et al (2009). More details of the facility and the experimental set-up can be found in Lee et al (2014). The obtained images are processed using an in-house PIV package. A final interrogation window size of $32 \times 32$ pixels is employed for these experiments, yielding a spatial resolution of approximately $50 \times 50$ viscous units. The lightsheet thickness is estimated at $1.5 \mathrm{~mm}$ or 50 viscous units. Further details on the PIV processing algorithms and calibration technique are documented in de Silva et al (2014).

Figure 5(a) shows viscous-scaled mean streamwise velocity profiles at four selected Reynolds numbers acquired from the towing plate PIV measurements $\left(R e_{\tau} \approx\right.$ 900, 1500, 2000 and 2500), together with the reference statistics from turbulent boundary layer simulation datasets of Eitel-Amor et al (2014) at comparable Reynolds numbers. For clarity of presentation, the data points are down-sampled and logarithmically spaced. Good collapse in the streamwise mean velocity profiles between the experimental and simulation results from $z^{+} \approx 100$ to the wake region is observed. However, comparisons of turbulence intensity profiles for the streamwise and wall-normal components shown in figure 5(b) and (c) show a large discrepancy between PIV and simulation. Hence, if careful assessment of these discrepancies is desired, the proposed estimation technique described in $\S 2$ can be performed.

Here, DNS volumes of the turbulent boundary layer at $R e_{\tau}=1500$ (Sillero et al, 2014) are volume filtered with matched $\Delta x^{+} \times \Delta y^{+} \times \Delta z^{+}$to the PIV measurements at four different Reynolds numbers (listed in table 1). A filter size of 50 viscous units is used in the spanwise direction to account for the laser sheet thickness. Thus, the magnitude of the small-scale missing 

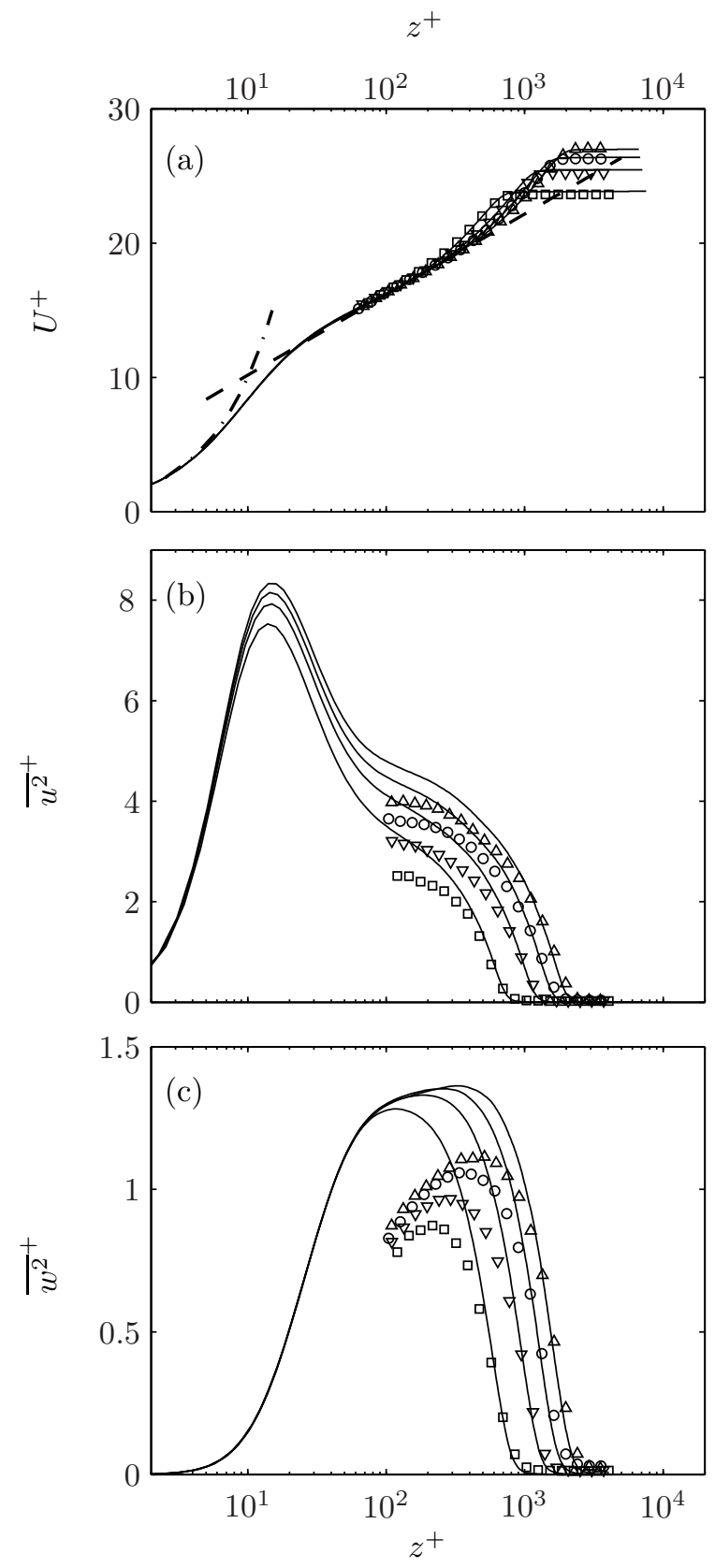

Fig. 5: Comparison between experimental results and the reference statistics of Eitel-Amor et al (2014) at matched Reynolds numbers. (a) Mean streamwise velocity, $U^{+}$. The dashed line shows $U^{+}=$ $(1 / 0.384) \ln \left(z^{+}\right)+4.17$. The dot-dashed line shows $U^{+}=$ $z^{+}$. (b) streamwise turbulence intensity, $\bar{u}^{+}$. (c) wallnormal turbulence intensity $\overline{w^{2}}$.

variance can be computed for the streamwise $\left(\left.\Delta \overline{u^{2}}\right|_{S R^{+}}\right)$ and wall-normal $\left(\left.\Delta \overline{w^{2}}\right|_{S R^{+}}\right)$components using equation (1). These missing energy profiles are then subtracted from the reference profiles from Eitel-Amor et al (2014) to produce the 'attenuated' estimation profiles
$\left(\left.\bar{u}^{2}\right|_{E s t}\right.$ and $\left.\left.{\overline{w^{2}}}^{+}\right|_{E s t}\right)$ purely due to the spatial averaging as shown in equation (2). Note that the statistics from Eitel-Amor et al (2014) are employed since the fully-resolved turbulence intensity profiles are available at matched Reynolds numbers to the current PIV experiments. The resulting estimation profiles for the streamwise and wall-normal components are shown using dashed lines in figure 6 and compared against the towing plate PIV experimental results (symbols). The grey lines in the plot represent the reference statistics. Figure 6 presents good agreement between the experimental and the estimated statistics throughout the boundary layer for both the streamwise and wallnormal components. Therefore, the discrepancy between the experimental and fully resolved turbulence intensities can now be attributed to the under-resolved smallscale energy contribution. The direct comparison between these profiles confirms the invariance of the innerscaled small-scale energy across Reynolds numbers, since the small-scale missing variance for both $u$ and $w$ components obtained from DNS datasets at $R e_{\tau} \approx 1500$ successfully estimate the spatial attenuations in Reynolds stresses for the range of Reynolds numbers considered. Though figure 6 only shows validations for ${\overline{u^{2}}}^{+}$and ${\overline{w^{2}}}^{+}$, the same technique has been applied to a further in-house PIV experiment to verify that the obtained spanwise variance $\bar{v}^{+}$is reasonable, with similarly convincing results.

To further assess the applicability of the estimation scheme in higher Reynolds numbers, planar PIV data of turbulent boundary layers at $R e_{\tau} \approx 6700$ from de Silva et al (2015) are used. This dataset has a spatial resolution of $75 \times 67 \times 75\left(\Delta x^{+} \times \Delta y^{+} \times \Delta z^{+}\right)$viscous units. Since fully resolved statistics from DNS at this Reynolds number are not currently available, well- but not fully-resolved measurements such as from HWA can be used to produce the estimated profile $\left.{\overline{u^{2}}}^{+}\right|_{E s t}$. Here we take single-normal hot-wire variance $\overline{u^{2}}{ }_{h w}$ from Marusic et al (2015) at the same Reynolds number, which measured with wire length of 22 viscous units. Therefore, we must first account for the missing energy due to the wire length to obtain $\left.\overline{u^{2}}\right|_{F R}$ profile. That is,

$$
\left.{\overline{u^{2}}}^{+}\right|_{F R}=\left.{\overline{u^{2}}}^{+}\right|_{h w}+\left.\Delta{\overline{u^{2}}}^{+}\right|_{l^{+}},
$$

where $\left.\Delta \bar{u}^{+}\right|_{l+}$ is its corresponding unresolved smallscale energy due to the wire length $l^{+}$. In this case, one could choose from any of the myriad correction schemes for single-normal hot-wires from existing literature for $\bar{u}^{2}$ (e.g. Smits et al, 2011; Chin et al, 2011). Alternatively, we can just modify our scheme with $\Delta y^{+}=l^{+}$ 

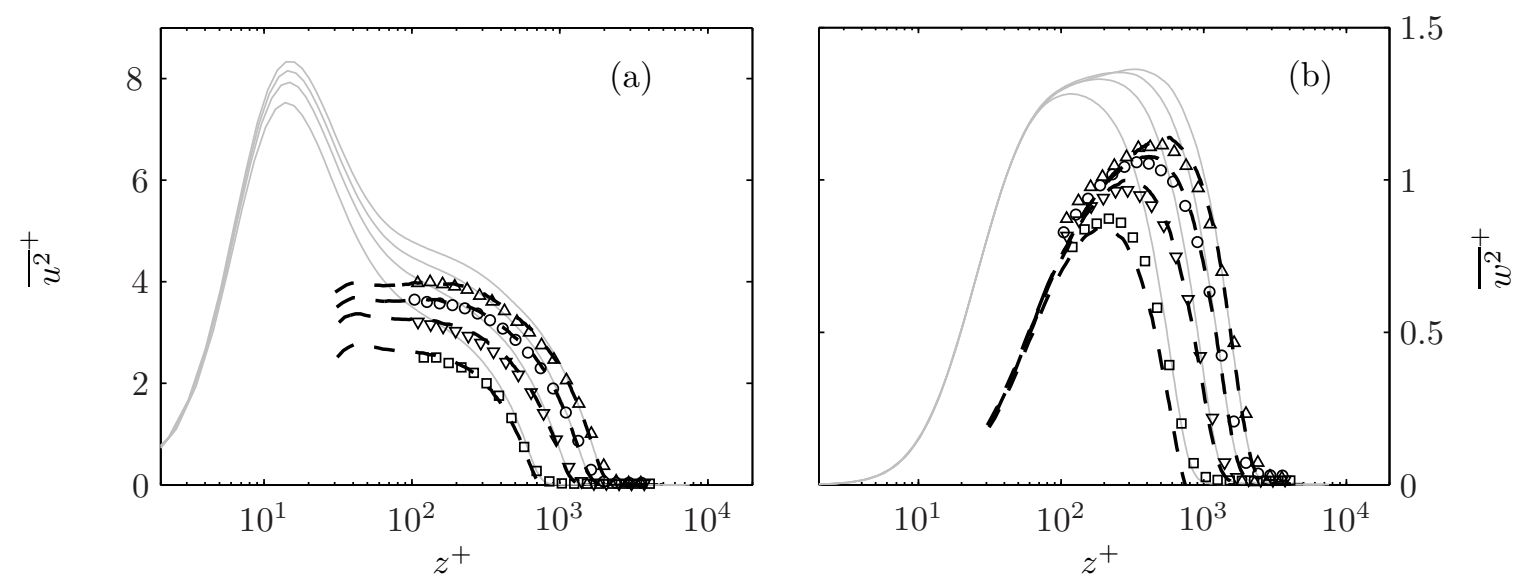

Fig. 6: Comparison between experimental and estimation technique results for (a) streamwise and (b) wall-normal turbulence intensity profiles across measured Reynolds numbers. Dashed lines indicate 'under-resolved' estimated statistics. Grey lines are the well-resolved correspondence of Eitel-Amor et al (2014).

and $\Delta x^{+}=\Delta z^{+}=0$ and filter low Reynolds number DNS fields to simulate the hot-wire measurement. Hence, subtracting the filtered from the unfiltered DNS gives $\left.\Delta{\overline{u^{2}}}^{+}\right|_{l^{+}}$. This then can be added to the measured $\bar{u}^{2}{ }_{h w}$ to produce a reference profile at this Reynolds number. In short, if we wish to compare the PIV to a better resolved measurement (such as HWA), we must account the missing energy from both measurements. Here, we use the current scheme and equation (3) to obtain a pseudo fully-resolved turbulent intensity profile at $R e_{\tau} \approx 6700$. And then the steps described in $\S 2$ are applied to compute the estimation profile (dashed line) as shown in figure 7 . The comparison between the estimation and the PIV profiles shows good collapse, indicating that the missing energy $\left(\left.\Delta{\overline{u^{2}}}^{+}\right|_{S R^{+}}\right)$acquired from the lower Reynolds number DNS volume provides a good approximation of missing small-scale contribution even at much higher Reynolds number. This result gives confidence that the proposed methodology can be applied to a wide range of Reynolds numbers.

The following section outlines a database of innerscaled small-scale missing energy profiles that we provide, for all three velocity components as a function of spatial resolution.

\section{Missing energy database for a turbulent boundary layer}

The steps to obtain the missing small-scale energy for a given spatial resolution by spatially filtering the DNS volumes are described in detail in $\S 2$. To summarise, to use this estimation tool, one requires access to full DNS volumes within which a spatial filter must be applied

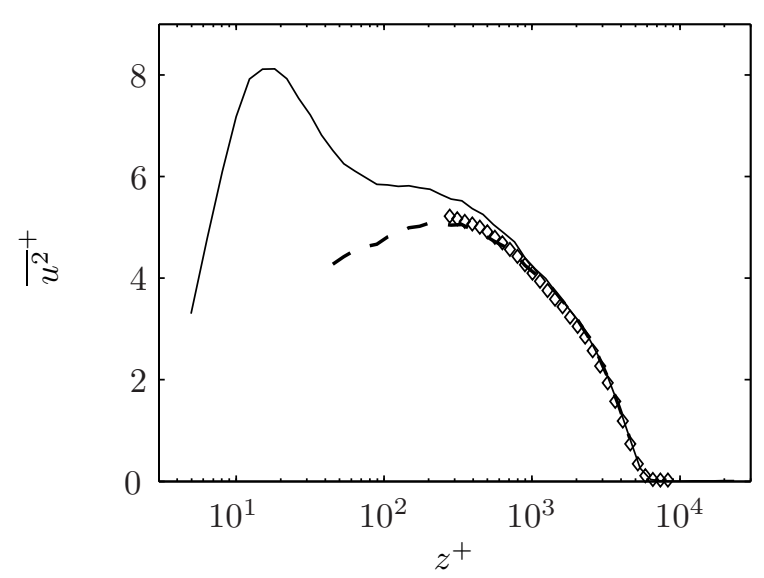

Fig. 7: Comparison of planar PIV, HWA experiments and estimation at $R e_{\tau} \approx 6700$ for $\bar{u}^{2}$. The solid line represents hot-wire data from Marusic et al (2015), the diamond symbol $(\diamond)$ indicates PIV data from de Silva et al (2015), and the dashed line shows the estimation obtained using the hot-wire data (using equation (3)).

with viscous scaled dimensions matched to the spatial resolution of the experiment. Here, we attempt to provide a database of missing small-scale energy profiles for $u, v$ and $w$ components across a reasonably inclu-

\begin{tabular}{ccccccc}
\multicolumn{7}{c}{ spatial filter sizes in viscous units } \\
\hline$\Delta x^{+}$ & 0 & 20.1 & 40.2 & 60.3 & 80.4 & 100.5 \\
$\Delta y^{+}$ & 0 & 22.8 & 45.6 & 68.4 & 91.2 & 114.0 \\
$\Delta z^{+}$ & 0 & 20 & 40 & 60 & 80 & 100 \\
\hline
\end{tabular}

Table 2: Inner-scaled filter sizes applied on the DNS volumes of a turbulent boundary layer (applied in all combinations). 

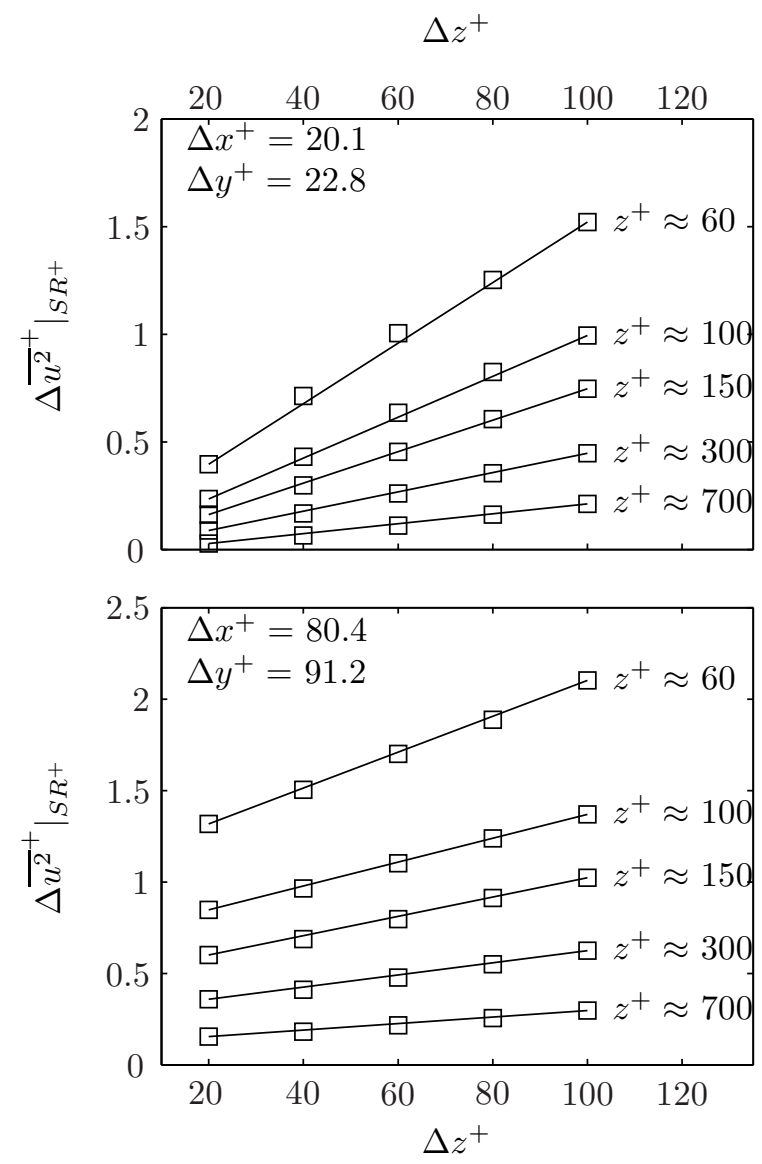

Fig. 8: Variation of the inner-scaled missing energy $\left.\Delta{\overline{u^{2}}}^{+}\right|_{S R^{+}}$with the spatial resolutions $\left(\Delta x^{+} \times \Delta y^{+} \times\right.$ $\left.\Delta z^{+}\right)$at several different fixed wall heights.

sive parameter space of encountered spatial resolutions. The aim here is to provide a convenient tool for estimating missing energy due to finite resolution, without each user requiring access to full DNS fields. The database is obtained by spatially filtering a turbulent boundary layer DNS volume of Sillero et al (2014) at $R e_{\tau} \approx 1500$ with various filter sizes. A total number of 216 different filter size combinations based on the spatial resolutions listed in table 2 are employed to generate the database. It should be noted that the filter size of zero in table 2 indicates the original DNS volumes based on an assumption that the simulation is fully resolved. Also, the largest filter size used in the database is approximately 100 viscous units $\left(\approx 0.07 \delta\right.$ at $\left.R e_{\tau}=1500\right)$ to minimise the filtering of any large-scale contribution in the current DNS datasets. If a higher Reynolds number database were available, this maximum filter size could be increased.

An interpolation method for computing the missing energy profiles for any spatial resolution (from unfiltered to approximately 100 viscous units in the $x, y$
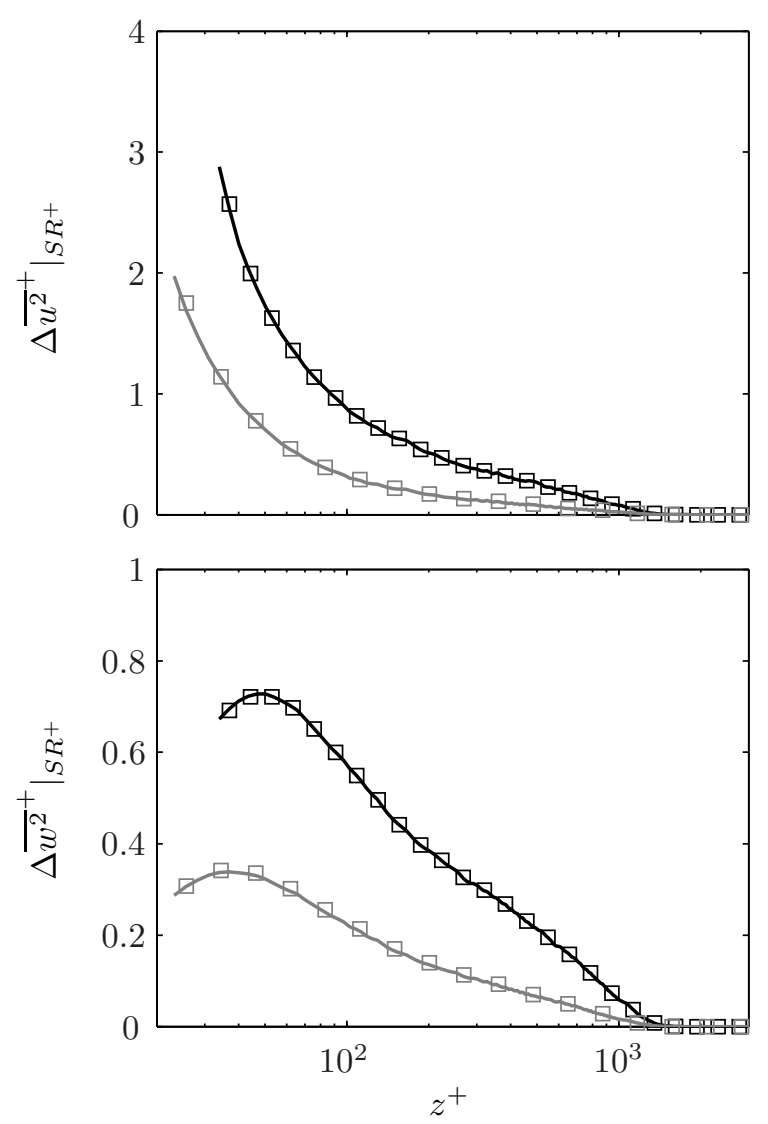

Fig. 9: Comparison of missing energy profiles for $u$ and $w$ components computed from filtered DNS (solid lines) and linear interpolation method (symbols) with the database for two different spatial resolutions. The spatial resolutions of $\Delta x^{+} \times \Delta y^{+} \times \Delta z^{+} \approx 54 \times 50 \times 55$ and $27 \times 23 \times 25$ are plotted in black and grey colours respectively.

and $z$ directions) is developed and a MATLAB script demonstrating this interpolation is included as Supplementary information. Examples of the variation of $\left.\Delta{\overline{u^{2}}}^{+}\right|_{S R^{+}}$with spatial resolutions at different fixed wall heights is shown in figure 8 . The data points indicate that an increase in the spatial filter size (in this case $\Delta z^{+}$) causes an approximately linear increase in the missing energy for a given fixed wall height. This suggests that the linear interpolation scheme across this database can be used to estimate a missing energy at a particular wall position for any given effective spatial resolution/interrogation volume. Figure 9 compares the missing energy profiles obtained by directly filtering the DNS volumes at a specific interrogation volume size and the results from linear interpolation method for two different spatial resolution examples for $u$ and $w$ components. A good collapse between the direct filtering (solid line) and the interpolated values (symbols) is observed 
throughout the boundary layer, suggesting that the provided database and the linear interpolation scheme are sufficient to estimate a missing energy profile for a given spatial resolution. Similar collapses are observed across the entire range of filter sizes and also for all velocity components $u, v$ and $w$. The missing energy profiles for the streamwise velocity component $\left.\Delta{\overline{u^{2}}}^{+}\right|_{S R^{+}}$(shown in figure 9) are reminiscent of the inverse $z^{+}$dependence of the unresolved energy discussed in Smits et al (2011). In their method, they provide a simple functional form to correct the attenuated streamwise variance. This functional form requires a pre-factor that considers 1-D averaging in the spanwise direction of the single-normal hot-wire length $l^{+}$. However, in the presence of 3-D volume averaging, formulating a functional form that involves $\Delta x^{+}, \Delta y^{+}$and $\Delta z^{+}$is not trivial. In addition, the missing energy profiles for the wallnormal component $\left.\Delta{\overline{w^{2}}}^{+}\right|_{S R^{+}}$shown in figure 9 do not seem to follow the inverse $z^{+}$dependence. Therefore, we choose to provide the missing-energy database as a look-up table. The merit of this database is that it covers the scenario of all three velocity components for any given averaging dimension.

This database and interpolation scheme also enable the missing energy from a single-point measurement such as a hot-wire sensor to be calculated. A single normal wire filters the data in a single direction (along the length of the sensor element $l^{+}$). Single normal wires are typically oriented along the spanwise direction $y$. Hence for this case, the database of missing energy and the interpolation method can be used, with a filter size of $0 \times l^{+} \times 0$ to estimate the missing energy due to this spatial resolution of the hot-wire $\left.\Delta{\overline{u^{2}}}^{+}\right|_{l^{+}}$. As shown in equation (3), $\left.\Delta{\overline{u^{2}}}^{+}\right|_{l^{+}}$can be added to a turbulence intensity profile from hot-wire experiments to generate a reference (unattenuated) profile at a high Reynolds number.

In this study, the missing energy database is developed from a DNS database of a developing turbulent boundary layer Sillero et al (2014). However, one would expect that for the streamwise component $u$, this method could also be applied to internal wall-bounded turbulent flows. It has been documented that the smallscale near-wall $u$ energy content for internal and external geometries are quite similar (Monty et al, 2009). However, differences have been noted between the broadband intensity profiles of internal and external geometries for $v$ and $w$ components (Jiménez and Hoyas, 2008). This suggests that for these components for internal geometries, a different missing energy database (based on channel DNS) would need to be formulated.

\section{Conclusions}

An estimation method that predicts the small-scale missing energy which is unresolved due to limited spatial resolution in PIV experiments is proposed. The missing energy is estimated using a lower Reynolds number DNS dataset and applied to higher Reynolds numbers dataset. The method is based on the Reynolds number invariance argument of the inner-scaled small-scale contribution observed by Hutchins et al (2009). Good agreement between the experimental results and estimation profiles are observed, showing that the estimation tool successfully computes the missing energy for given spatial resolutions over a wide range of Reynolds numbers. A comparison between estimation and experimental results can be used to diagnose whether PIV statistics are beset by some wider issue, and also provides a sanity check of the expected degree of spatial attenuation. For convenience, a database covering the parameter space of likely interrogation volumes is computed, and a linear interpolation tool is provided that enables the convenient calculation of missing energy for PIV practitioners without the need to access DNS data. Throughout this study, we are limited to investigating 2-dimensional streamwise/wall-normal PIV planes in a turbulent boundary layer based on the experimental datasets. However, this tool can be applicable to other configurations of PIV such as tomographic PIV or wall-parallel plane or cross-stream plane.

\section{Acknowledgments}

The authors gratefully acknowledge the financial support of the Australian Research Council. The authors also wish to thank Dr J. A. Sillero and Professors J. Jiménez and R. D. Moser for making the DNS dataset publicly available.

\section{Supplementary information}

For a turbulent boundary layer, a database of the missing energy profiles for $u, v$ and $w$ components with various spatial resolutions (listed in table 2) is provided as supplementary information. This database is in the form of a . mat file (SR_MissingEnergy_DB. mat) which contains missing $u, v$ and $w$ energy (SR_u_DB, SR_v_DB, $\left.\mathrm{SR}_{-}{ }_{-} \mathrm{DB}\right)$ in the form of $4 \mathrm{D}$ matrices $(1000,6,6,6)$ which have data pertaining to $\left(z^{+}, \Delta x^{+}, \Delta y^{+}, \Delta z^{+}\right)$. An example MATLAB function below provides an example of how this database can be used to estimate the missing energy due to a given spatial resolution. SRdiff is the missing energy $\left.\Delta{\overline{u^{2}}}^{+}\right|_{S R^{+}}$(or the other components) 
estimated for a given resolution $\left(\mathrm{SR}_{-} \mathrm{x}, \mathrm{SR} \_\mathrm{y}, \mathrm{SR}_{-} \mathrm{z}=\right.$ $\Delta x^{+}, \Delta y^{+}, \Delta z^{+}$) at given wall heights (zexp which is a vector of $z^{+}$locations at which SRdiff is required).

\section{References}

Adrian RJ, Westerweel J (2011) Particle Image Velocimetry. Cambridge University Press, United Kingdom

Baidya R (2016) Multi-component velocity measurements in turbulent boundary layers. Ph.D. thesis, The University of Melbourne

Buxton ORH, Laizet S, Ganapathisubramani B (2011) The effects of resolution and noise on kinematic features of fine-scale turbulence. Experiments in Fluids 51:1417-1437

Chauhan KA, Monkewitz PA, Nagib HM (2009) Criteria for assessing experiments in zero pressure gradient boundary layers. Fluid Dynamics Research 41:021,404

Chin C, Hutchins N, Ooi A, Marusic I (2011) Spatial resolution correction for hot-wire anemometry in wall turbulence. Experiments in Fluids 50:1443-1453

Chin C, Philip J, Klewicki J, Ooi A, Marusic I (2014) Reynolds-number-dependent turbulent inertia and onset of $\log$ region in pipe flows. Journal of Fluid Mechanics 757:747-769

Eitel-Amor G, Örlü R, Schlatter P (2014) Simulation and validation of a spatially evolving turbulent boundary layer up to $R e_{\theta}=8300$. International Journal of Heat and Fluid Flow 47:57-69

Hutchins N, Nickels T, Marusic I, Chong M (2009) Hotwire spatial resolution issues in wall-bounded turbulence. Journal of Fluid Mechanics 635:103-136

Jiménez J, Hoyas S (2008) Turbulent fluctuations above the buffer layer of wall-bounded flows. Journal of Fluid Mechanics 611:215-236

Johansson AV, Alfredsson PH (1983) Effects of imperfect spatial resolution on measurements of wallbounded turbulent shear flows. Journal of Fluid Mechanics 137:409-421

Kähler CJ, Scholz U, Ortmanns J (2006) Wall-shearstress and near-wall turbulence measurements up to single pixel resolution by means of long-distance micro-PIV. Experiments in Fluids 41:327-341

Lavoie P, Avallone G, Gregorio FD, Romano GP, Antonia RA (2007) Spatial resolution of PIV for the measurement of turbulence. Experiments in Fluids 43:39-51

Lee JH, Kwon YS, Monty JP, Hutchins N (2014) Timeresolved PIV measurement of a developing zero pressure gradient turbulent boundary layer. In Proc. 18th Australasian Fluid Mech. Conference.
Ligrani P, Bradshaw P (1987) Spatial resolution and measurement of turbulence in the viscous sublayer using subminiature hot-wire probes. Experiments in Fluids 5:407-417

Marusic I, Chauhan KA, Kulandaivelu V, Hutchins N (2015) Evolution of zero-pressure-gradient boundary layers from different tripping conditions. Journal of Fluid Mechanics 783:379-411

Monty JP, Hutchins N, Ng H, Marusic I, Chong MS (2009) A comparison of turbulent pipe, channel and boundary layer flows. Journal of Fluid Mechanics 632:431-442

Ng HCH, Monty JP, Hutchins N, Chong MS, Marusic I (2011) Comparison of turbulent channel and pipe flows with varying Reynolds number. Experiments in Fluids 51:1261-1281

Philip J, Baidya R, Hutchins N, Monty JP, Marusic I (2013a) Spatial averaging of streamwise and spanwise velocity measurements in wall-bounded turbulence using $\vee$ - and $\times$ - probes. Measurement Science and Technology 24:115,302

Philip J, N Hutchins, Monty JP, Marusic I (2013b) Spatial averaging of velocity measurements in wallbounded turbulence: single hot-wires. Measurement Science and Technology 24:115,301

Saikrishnan N, Marusic I, Longmire EK (2006) Assessment of dual plane PIV measurements in wall turbulence using DNS data. Experiments in Fluids 41:265278

Segalini A, Örlü R, Schlatter P, Alfredsson PH, Rüedi JD, Talamelli A (2011) A method to estimate turbulence intensity and transverse Taylor microscal in turbulent flows from spatially averaged hot-wire data. Experiments in Fluids 51:693-700

Segalini A, Bellani G, Sardina G, Brandt L, Variano EA (2014) Corrections for one- and two-point statistics measured with coarse-resolution particle image velocimetry. Experiments in Fluids 55:1739

Sillero JA, Jiménez J, Moser RD (2014) Two-point statistics for turbulent boundary layers and channels at Reynolds numbers up to $\delta^{+} \approx 2000$. Physics of Fluids 26:105,109

de Silva CM, Baidya R, Marusic I (2012) Assesment of tomographic PIV using experimental and DNS data in turbulent channel flows. $16^{\text {th }}$ Int Symp on Applications of Laser Techniques to Fluid Mechanics, Lisbon, Portugal, 09-12 July

de Silva CM, Gnanamanickam EP, Atkinson C, Buchmann NA, Hutchins N, Soria J, Marusic I (2014) High spatial range velocity measurements in a high Reynolds number turbulent boundary layer. Physics of Fluids 26(2):025,117 
de Silva CM, Squire DT, Hutchins N, Marusic I (2015)

Towards capturing large scale coherent structures in boundary layers using particle image velocimetry. Proceedings of the 7th Australian Conference on Laser Diagnostics in Fluid Mechanics and Combustion

Smits A, Monty J, Hultmark M, Bailey SCC, Hutchins N, Marusic I (2011) Spatial resolution correction for wall-bounded turbulence measurements. Journal of Fluid Mechanics 676:41-53

Suzuki Y, Kasagi N (1992) Evaluation of hot-wire measurements in wall shear turbulence using a direct numerical simulation database. Experimental Thermal and Fluid Science 5:69-77

Worth NA, Nickels TB, Swaminathan N (2010) A tomographic PIV resolution study based on homogeneous isotropic turbulence DNS data. Experiments in Fluids 49:637-656

Wyngaard JC (1968) Measurement of small-scale turbulence structure with hot-wires. Journal of Physics E: Scientific Instruments 1:1105-1108 
function SRdiff = SR_estimation (zexp,SR_x,SR_y,SR_z, vel_comp)

$\%$ Estimation tool that calculates missing energy due to a given spatial

$\%$ resolution of an experiment

$\%$ Authors: J. H. Lee, Kevin, J. P. Monty and N. Hutchins, The University of Melbourne

\% Date: 29-February-2016

$\%$ INPUTS

$\%$ zexp = wall-normal $(z)$ position in viscous units for experiments

$\% \mathrm{SR}_{-} \mathrm{x}=$ spatial resolution in viscous units in the streamwise direction

$\% \mathrm{SR}_{-} \mathrm{y}=$ spatial resolution in viscous units in the spanwise direction

$\% \mathrm{SR}_{-} \mathrm{z}=$ spatial resolution in viscous units in the wall-normal direction

$\%$ vel_comp $=1$ - missing energy for u component (default)

$\% \quad 2$ - missing energy for $\mathrm{v}$ (spanwise) component

$\% \quad 3$ - missing energy for $w$ (wall-normal) component

$\%$

$\%$ OUTPUTS

$\%$ SRdiff = linearly interpolated missing energy profile (inner-normalised) throughout

$\%$ a given wall position for a given spatial resolution of an experiment

$\%$

$\%$ e.g if one wants to estimate the missing energy profile of u component

$\%$ for a spatial resolution of $50 \times 50 \times 50$ ( $\mathrm{xi} \times \mathrm{yi} \times \mathrm{zi}$ ) in viscous unit,

$\%$ uvardiff $=$ SR_estimation(zplus_exp, $50,50,50,1)$;

$\%$

$\%$ e.g if one wants to estimate the missing energy profile of u component

$\%$ for a single-normal hotwire with a sensor length of 22 viscous wall units

$\%$ oriented in the spanwise direction.

$\%$ uvardiff_lplus = SR_estimation(zplus_exp, $0,22,0,1)$;

load ('SR_MissingEnergy_DB.mat')

if nargin $<5$ || vel_comp $==1$;

SR_DB $=\mathrm{SR}_{-} \mathrm{u}_{-} \mathrm{DB}$;

elseif vel_comp $==2$;

SR_DB $=$ SR_V_DB; $_{-}$

elseif vel_comp $==3$;

SR_DB $=$ SR_w_DB;

end

if $\mathrm{SR}_{-} \mathrm{z}==0$

[zplus_exp, $\operatorname{xexp}, \operatorname{yexp}]=\operatorname{ndgrid}\left(z \exp , \mathrm{SR}_{-} \mathrm{x}, \mathrm{SR}_{-} \mathrm{y}\right)$;

SRdiff = interpn(zplus_DB $\left.(:,:,:, 1), x_{-} D B(:,:,:, 1), y_{-} D B(:,:,:, 1), S_{-} D B(:,:,:, 1), z p l u s \_\exp , x \exp , y e x p\right)$ else

[zplus_exp, xexp, yexp,zexp] = ndgrid (zexp,SR_x,SR_y, SR_z);

SRdiff $=$ interpn(zplus_DB, x_DB,y_DB,z_DB, SR_DB, zplus_exp, $x \exp , y \exp , z \exp )$;

end

end 


\section{University Library}

\section{- M M N E R VA A gateway to Melbourne's research publications}

Minerva Access is the Institutional Repository of The University of Melbourne

Author/s:

Lee, JH;Kevin,;Monty, JP;Hutchins, N

Title:

Validating under-resolved turbulence intensities for PIV experiments in canonical wallbounded turbulence

Date:

2016-08-01

\section{Citation:}

Lee, J. H., Kevin, , Monty, J. P. \& Hutchins, N. (2016). Validating under-resolved turbulence intensities for PIV experiments in canonical wall-bounded turbulence. EXPERIMENTS IN FLUIDS, 57 (8), https://doi.org/10.1007/s00348-016-2209-6.

Persistent Link:

http://hdl.handle.net/11343/282553 